\title{
NETWORKS FOR HELIOSEISMIC OBSERVATIONS
}

\author{
Frank Hill \\ National Solar Observatory \\ National Optical Astronomy Observatories \\ Tucson, Arizona 85726 USA
}

\begin{abstract}
Helioseismology from a single ground-based observatory is severely compromised by the diurnal rising and setting of the Sun. This causes sidelobes to appear in the helioseismic power spectrum at multiples of $\pm 11.57 \mu \mathrm{Hz}$ from each solar line, contaminating the spectrum and rendering mode identification and frequency measurement extremely difficult. The difficulty can be overcome in three ways - observing from a fully sunlit orbit in space, observing from the Polar regions, or observing with a network of stations placed around the Earth. This paper discusses the networks that are either currently in operation or being planned. These include the Global Oscillation Network Group (GONG) project, the Birmingham network, the IRIS network of the University of Nice, and the SCLERA network of the University of Arizona. The scientific objectives and instrumentation of these networks are briefly described. Theoretical predictions for network performance are compared with actual results. The problem of merging simultaneous data from multiple instruments is discussed, as well as the relationship of the networks with the helioseismology experiments on the SOHO space mission.
\end{abstract}

\section{The Need For Continuous Data - The Scientific Issues}

Helioseismology, the study of solar oscillations, is a powerful tool for probing the solar interior. The oscillations are global and permeate the entire Sun, thus their properties are sensitive to the Sun's internal structure. Careful measurements of the frequencies, amplitudes, and lifetimes of the oscillations provide information on the internal sound speed, temperature, rotation rate, composition, opacity, convective flow patterns, and magnetic field. Comparison of the observed frequencies with theoretical predictions derived from solar models helps to test potential explanations for the observed deficiency of solar neutrinos such as the cosmion or WIMP model (e.g. Faulkner, Gough and Vahia 1986; Bahcall and Ulrich 1988; see also several papers in these proceedings and contributed posters in the Solar Physics volume).

The most useful measurements are the frequencies of the oscillations as a function of their spherical harmonic "quantum numbers" $-\ell$, the spherical harmonic degree, $m$, the azimuthal degree, and $n$, the radial order. It is relatively simple to compute these frequencies from theoretical solar models, and the observed frequencies can also be directly combined via inverse techniques to infer the depth dependence of physical conditions (see Gough 1985 for a review of inverse techniques). Frequencies are also the most easily measurable property of the oscillations. The temporal length of the sequence of observations, $T$, and the precision with which the frequencies $v$ can be determined, $\Delta v$, are simply related: $\Delta v=1 / T$. Thus longer time strings are always the goal of the observational helioseismologist. Unfortunately for him or her, the Earth rotates and the Sun sets every day, placing periodic gaps in the sequence of

\footnotetext{
"Operated by the Association of Universities for Research in Astronomy, Inc. under contract with the National Science Foundation.
} 
observations.

This is especially unfortunate because observational time strings of many days, months, or even years are essential for helioseismic discrimination between different models of internal solar structure. As an example, the solar surface rotation period varies from about 28 days at the equator to 32 days at the pole. The rotation changes the frequency of the modes by an amount proportional to their $m$-value (the number of wavelengths around the equator of the coordinate system); the constant of proportionality is approximately the inverse of the rotation period. In order to resolve these shifts, one must have a frequency resolution of twice the shift, implying a minimum observational duration of two rotation periods or about 60 days. Even longer time strings are needed to investigate solar cycle changes that may occur over an 11-year period.
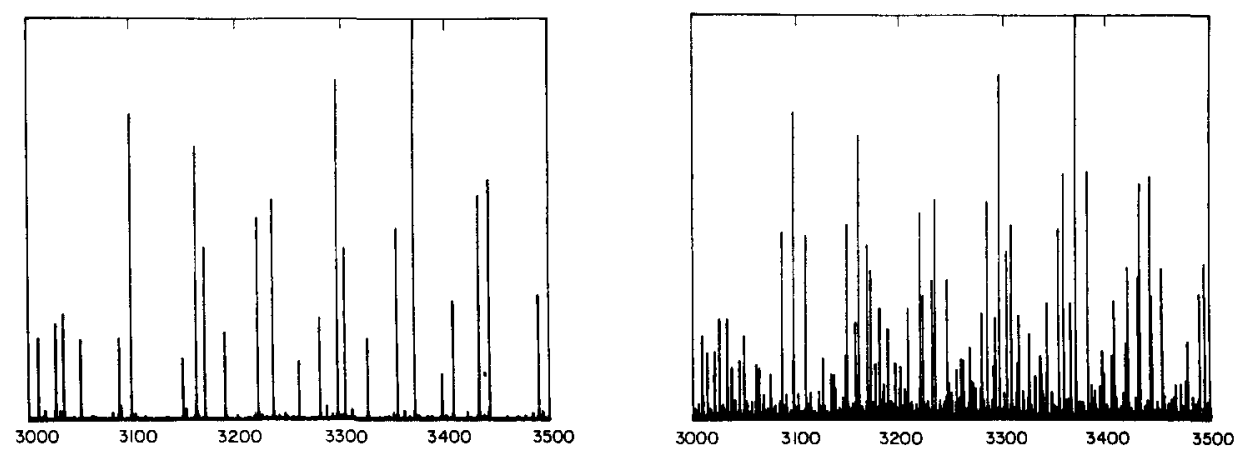

FIGURE 1. Left: The 3000 to $3500 \mu \mathrm{Hz}$ band of a simulated solar spectrum as might be obtained from continuous observations covering one year. Right: The same portion of the simulated spectrum as might be obtained at a single mid-latitude site experiencing the day-night cycle and typical weather patterns.

Fourier analysis shows that when two functions are multiplied together in the temporal domain, their transforms are convolved in the frequency domain. For helioseismology, the two functions are the time series of solar data and a time series of ones or zeroes representing the times of data (ones) or no data (zeroes) called the window function. Thus, in the frequency domain we obtain the real solar spectrum convolved with the spectrum of the window. The window function has a regular diurnal periodic structure, thus its spectrum consists of a strong peak at a frequency of $1 / \mathrm{day}$, or $11.57 \mu \mathrm{Hz}$, along with its harmonics at integral multiples of the fundamental 1/day frequency. These peaks are called diurnal sidelobes; the fundamental diumal sidelobe typically has an amplitude that is $60 \%$ of the amplitude of the zero frequency component of the window spectrum. Since the solar spectrum and the window spectrum are both nearly sets of discrete delta functions, the convolution of the window spectrum with the solar spectrum results in a replica of the window spectrum appearing symmetrically around every solar spectral line, as shown in Figure 1. As can be seen in Figure 1, there are many instances where a solar line overlies a diurnal sidelobe, greatly compromising mode identification and frequency measurements. An example of a window function is shown in Figure 2, where the window as actually observed at Tucson is displayed on a grid representing one hour increments, with black representing times of no observations due to either weather or nighttime. Nighttime is clearly visible as the prominent black swaths across the diagram. The first $60 \mu \mathrm{Hz}$ of the power spectrum of the window in Figure 2 is shown in Figure 3. The fundamental and the first four harmonics of the diumal sidelobes are clearly visible, as is a noise background.

Several data processing schemes to eliminate the effects of the diurnal sidelobes have been investigated. Radio astronomers have long faced the problem of unwanted sidelobes, and have developed an iterative peak subtraction algorithm known as CLEAN. It has been used to search for solar gravity modes (Delache and Scherrer 1983; Scherrer 1984), and to remove the sidelobes from $p$-mode spectra 


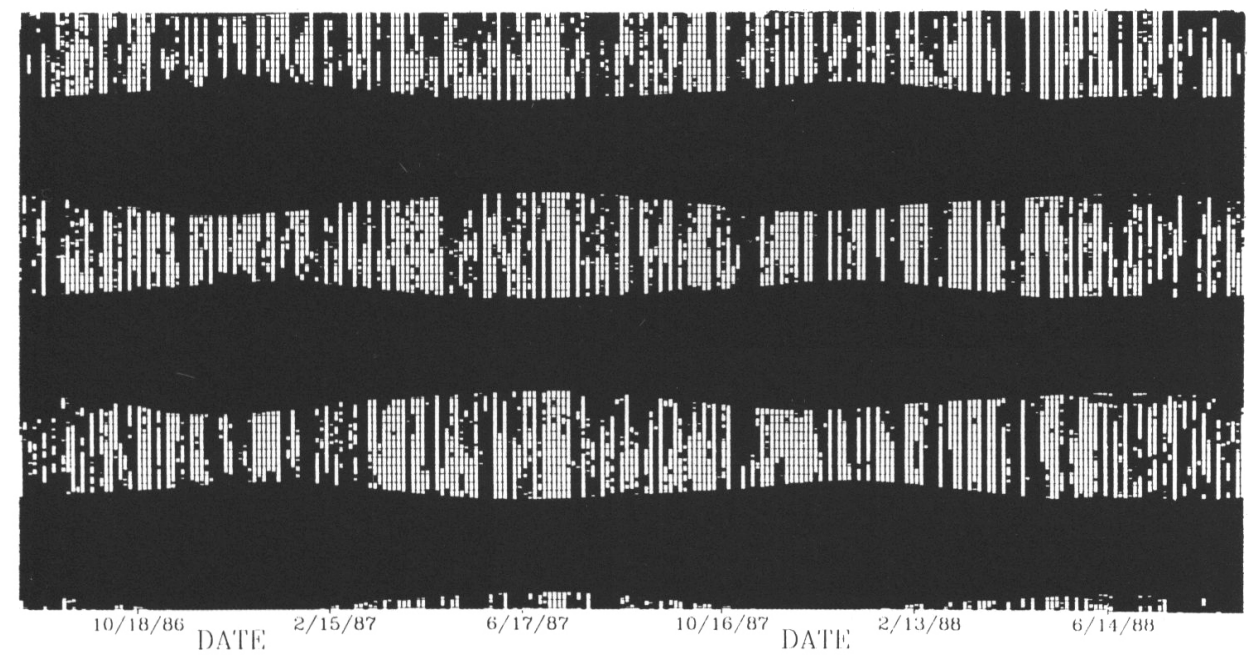

FIGURE 2. An example of an actual window function observed at Tucson, Arizona. Each box in the grid represents one hour of observations, with the hours arranged in columns of 72 hour or 3 day intervals. Times of no sunlight are indicated by black areas. Night and its seasonal variation is clearly visible as the three prominent black swaths across the figure.

(Duvall and Harvey 1984; Henning and Scherrer 1986). In the context of helioseismology, the CLEAN algorithm can misidentify a sidelobe as a solar frequency. Another method rearranges the data to fill in the daily gaps, but this reduces the frequency resolution of the spectrum (Kuhn 1982, 1984). Linear iterative deconvolution techniques tend to increase the noise in the spectra, while nonlinear spectral deconvolution (Connes and Connes 1984) assumes that the actual signal is band limited and that power outside the band comes from the gaps. This assumption is not met by the solar oscillations (Scherrer, 1986). Maximum entropy has been used to extrapolate data into gaps (Fahlman and Ulrych 1982), and this method has been studied in the context of helioseismology (Brown and Christensen-Dalsgaard 1985, unpublished). They found that solar spectra can be reliably recovered if the filling factor, or duty cycle, is greater than 0.8 , and the signal-to-noise ratio is larger than 100 . Finally, Bayesian probability techniques have been used to combine all available $a$ priori information along with the observed power spectra to extract and indentify the modes (Morrow and Brown 1988; Brown 1988). This method has also proven to be capable of misidentifying modes. One must draw the conclusion that, so far, no technique is available that will allow the reliable elimination of the diurnal sidelobes from spectra obtained at a single ground-based site. We must look to other observing strategies to eliminate the daily gaps as much as possible during the observing process itself.

\section{Strategies For Obtaining Continuous Data}

There are three basic strategies that enable helioseismologists to obtain nearly continuous data. These strategies are observing at the polar regions, observing from space, or observing from a network of ground-based stations. The strategies are summarized in Table 1. All of the strategies face environmental and logistical challenges, and produce large data flows if they involve observations with moderate to high spatial resolution.

The Sun stays above the horizon for six months at a time at the polar regions, thus observations from these regions are capable of providing more than the 14 continuous hours available from lower 


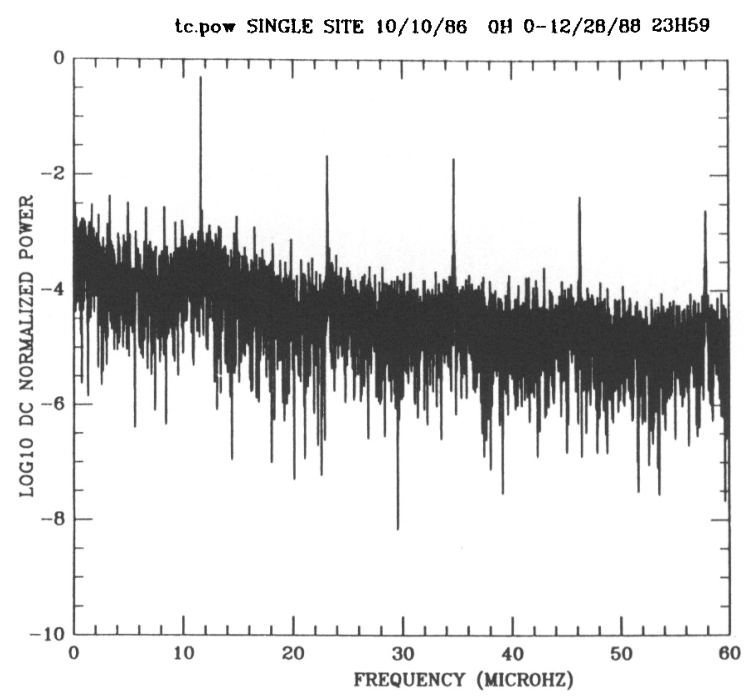

FIGURE 3. The first $60 \mu \mathrm{Hz}$ of the power spectrum of the window function in Figure 2. The power has been normalized by the power in the zero-frequency or DC component and the logarithm taken. The fundamental and first four harmonics of the diumal sidelobes are clearly visible. It is the reduction of these sidelobes that is the goal of the network concept.

latitude single sites. In practice, the presence of a continental land mass at the South pole and the associated infrastructure makes it the pole of choice for observational astronomy. Several helioseismology experiments have been carried out at the South pole, including non-imaging observations (Grec, Fossat and Pomerantz 1983); diameter measurements (Stebbins and Wilson 1983), and imaged observations (Jefferies et al. 1988b). There are three advantages to a South pole experiment. First, since only a single instrument is involved, there is no need to merge simultaneous data sets. Second, the cost of the experiment is substantially lower than that of the other two strategies. Finally, it is possible to recover from instrumental failure, although it is rather more difficult than at sites at lower latitudes. The disadvantages are twofold: First, the weather patterns at the South pole place a limit on the uninterrupted duration of the observations of about seven days, far short of the sixty needed to cleanly separate modes with adjacent values of $m$. Secondly, the presence of the Earth's atmosphere degrades the spatial response of the solar power spectra, especially at higher values of $\ell$ (F. Hill et al. 1984). The logistical challenge consists of the remoteness of the site and the harshness of the environment.

A space platform can be placed in a fully sunlit orbit about the Earth or around a Lagrangian point. Various studies have been made of space-borne helioseismic observations (e.g. Newkirk 1980, Noyes and Rhodes 1984), and the European Space Agency will be launching the SOHO mission in the mid 1990s (Domingo 1988; see also the paper by Bonnet in these Proceedings). There are two advantages to the space-borne observations: First, there is naturally no degradation of the observations from atmospheric seeing. Secondly, no data merging is required for a single instrument. Three disadvantages exist: First, there is little chance for recovery from an instrumental failure, especially for a spacecraft in orbit around the $L_{1}$ point. Second, telemetry bandwidth limitations render it impossible to transmit the entire data set from a high-resolution imaging experiment, thus requiring on-board data processing and forcing a selection of data parameters. Telemetry ground stations are typically three-site networks which are vulnerable to single-site instrumental failures. Furthermore, they may not provide 24 -hour coverage in all seasons. Finally, a space experiment is monetarily the most expensive method of obtaining nearly continuous data. The lifetime and hence the frequency resolution of a space experiment is limited by the expendable supplies such as propellant, and the logistical challenges consist of placing the vehicle in orbit, and coping 
Table 1

A Comparison of Observing Strategies for

Obtaining Continuous Helioseismic Data

\begin{tabular}{|lll|}
\hline \multicolumn{1}{|c|}{ Strategy } & \multicolumn{1}{c|}{ Advantages } & \multicolumn{1}{c|}{ Disadvantages } \\
\hline \hline $\begin{array}{l}\text { South Pole } \\
\begin{array}{l}\text { Sun always above horizon dur- } \\
\text { ing Austral summer }\end{array}\end{array}$ & $\begin{array}{l}\text { - No data merging required } \\
\text { - Inexpensive }\end{array}$ & $\begin{array}{l}\text { - Limited frequency resolution } \\
\text { - Terrestrial atmospheric de- } \\
\text { gradation }\end{array}$ \\
\hline $\begin{array}{l}\text { Space platform } \\
\begin{array}{l}\text { Can be placed in fully sunlit } \\
\text { orbit }\left(e . g . \mathrm{L}_{\mathbf{1}}\right)\end{array}\end{array}$ & - No atmospheric degradation & - No failure recovery \\
\hline $\begin{array}{l}\text { Network } \\
\text { Longitude coverage and redun- } \\
\text { dancy reduces diurnal cycle } \\
\text { and impact of weather and } \\
\text { failures }\end{array}$ & $\begin{array}{c}\text { - Failure recovery possible } \\
\text { tion }\end{array}$ & - Telemetry limitations \\
\hline
\end{tabular}

with the radiation and thermal environment.

A network of identical observing stations redundantly distributed in longitude reduces the diurnal sidelobes as well as the impact of weather and instrumental failures. The optimal number of sites for a network is six, distributed in longitude so that at least two stations are potentially observing at all times. Models predict that such a network should provide observations with a duty cycle of about $93 \%$ (F. Hill and Newkirk 1985); as discussed in section 4, this agrees well with actual results (e.g. F. Hill and the GONG Site Survey Team 1988). With a duty cycle this high, and assuming that the instrument is welldesigned and built with a signal-to-noise ratio greater than 100 , then the remaining gaps should be easily filled using the maximum entropy method. The advantages of a network are twofold: First, unlimited frequency resolution is available in principle. Secondly, it is possible to recover from instrumental failures. There are two disadvantages: The existence of multiple sites observing simultaneously requires that the data be merged, a problem for which there is currently little previous experience. Secondly, the observations are degraded by seeing and transparency gradients in the Earth's atmosphere. The logistical challenge consists of manufacturing a number of identical instruments and engineering them to withstand almost the entire range of climates found on the Earth. In addition, the international aspect provides the opportunity to sample the customs of many nations. The expense of an imaging network is moderate, being substantially less than a space experiment, but more than a South pole experiment.

\section{Summary Of Current And Future Networks}

Four major networks are either currently in partial operation, or being planned. None of these networks have yet reached full operational status of six or more sites. In addition, there have been a number of two-site networks with limited lifetimes. 
Historically, the original helioseismology network is the Birmingham Solar Seismology Network, with headquarters at the University of Birmingham in the United Kingdom (Claverie et al. 1984; Aindow et al. 1988; Elsworth et al. 1988b). It was proposed in 1975, and funded for a full six stations in 1987. The first station was installed at Izaña, Tenerife in the Spanish Canary Islands off the western coast of Africa in 1975. A second station was installed in 1981 at Haleakala, Hawaii, and a third automatic station began operations in 1985 at Carnarvon, Western Australia. A fourth station will be installed in 1989 at Sutherland, South Africa. Two additional sites will be selected and installed at a future time. The Birmingham Network instrumentation consists of a non-imaging potassium optical resonance spectrometer. Since the experiment is non-imaging, the observations are sensitive only to oscillations with $0 \leq \ell \leq$ 3. Scientific objectives of the experiment have included the measurement of line widths (Isaak 1986; Elsworth et al. 1988a); solar internal structure (Claverie et al. 1979); rotational splitting (Jefferies et al. 1988a); $g$ modes (Isaak et al. 1984; van der Raay 1988; Garcia, Pallé and Roca Cortés 1988); solar cycle frequency changes (Isaak et al. 1988; Jiménez et al. 1988a, Palle, Régulo and Roca Cortés 1989); and the identification of modes in the low $\ell$ portion of the solar spectrum (Palle et al. 1986; Anguera et al. 1989).

A second non-imaging network is being developed and administered at the Université de Nice in France. This network is known as IRIS (Installation d'un Réseau International de Sismologie Solaire or International Research on the Interior of the Sun) (Fossat 1988). This network was proposed in 1983 and funded for seven stations in 1984. A prototype instrument was operated at La Silla, Chile from May 1986 to the Spring of 1987. Three instruments are currently operating at Stanford, California (operations began in 1987), Kumbel, Uzbekistan, U. S. S. R. (1988), and l'Oukaimeden, Morocco (1988). Two more instruments are scheduled to come on-line in 1989 at La Silla, and Izaña. The final two instruments will be installed in 1990 at Learmonth, Western Australia, and Haleakala. The operation of the IRIS network is scheduled to continue for one full solar cycle of 11 years, ending in 2001. The IRIS instrument is similar to the Birmingham device, but uses sodium instead of potassium (Grec, Fossat and Vemin 1976). The full-disk observations are sensitive to oscillations with $0 \leq \ell \leq 3$. The Nice group has been active with a single instrument for some time. Scientific goals of the IRIS project include solar cycle frequency changes (Fossat et al. 1987; Gelly, Fossat and Grec 1988); g modes (Fossat et al. 1988; Provost and Berthomieu 1988); frequency and amplitude measurements (Grec, Fossat and Pomerantz 1983); and asteroseismology (Gelly, Grec and Fossat 1986; Gelly et al. 1988b; Schmider et al. 1988). The Nice group has also observed at the South pole (Gelly et al. 1988a).

Measurements of the solar diameter have also been used to search for solar oscillations (H. Hill 1984). Although the results of such studies are controversial (e.g. Yerle 1988), a network of instruments to obtain the measurements is being planned. The SCLERA (Santa Catalina Laboratory for Experimental Relativity and Astrometry) network is being developed at the University of Arizona in Tucson. Two sites at Tucson, Arizona and Yunnan Observatory, Kunming, China, have been selected, and negotiations are underway for a third site in the U.S.S.R. Other sites will be determined at a future time. The instrumentation will comprise a combination of diameter measurements and broad-band photometry of the central portion of the disk. The scientific objectives of the group have included the gravitational quadrupole moment (H. Hill, Bos and Goode 1982); the 160-minute oscillation (H. Hill, Tash and Padin 1986); $g$ modes (H. Hill, Gao and Rosenwald 1988); internal rotation (H. Hill et al. 1986); and the neutrino problem (H. Hill 1986).

The final major network is perhaps the most ambitious. The Global Oscillation Network Group (GONG) project is planning to place six imaging instruments around the world in 1992 (Harvey, Kennedy and Leibacher 1987; Harvey et al. 1988a). The network was proposed in 1984 and funded in 1985. Guided by models (F. Hill and Newkirk 1985), a site survey is underway to select the six sites from fourteen candidates (Fischer et al. 1986; F. Hill and the GONG Site Survey Team 1988). The candidate sites are Cerro Tololo and Las Campanas, Chile; Izaña; l'Oukaimeden; Riyadh, Saudi Arabia; Udaipur, India; Urumqi, China; Learmonth; Haleakala, Mauna Kea and Mauna Loa, Hawaii; and Big Bear, Yuma and Tucson, U.S.A. The site survey has been underway since 1985 , and site selection is currently planned for 1991. Deployment of the network is now scheduled for 1992, with observations starting in 1993 and ending in 1996. The instrument is under development and comprises a Fourier tachometer design using a 
Michelson interferometer and a Lyơt prefilter (Harvey and the GONG Instrument Development Team 1988). The detector nominally will be a $256 \times 256 \mathrm{CCD}$, and the instrument will feature laser calibration, automatic operation, and Exabyte cartridge data storage. The amount of data produced by the experiment will total approximately three terabytes, necessitating careful planning of the data reduction system (Kennedy and Pintar 1988; Pintar and the GONG Data Reduction Team 1988). The project is open to all helioseismologists, and so its scientific objectives cover the entire range of helioseismology. With the current detector configuration, oscillations with $0 \leq \ell \leq 150$ will be observable. Scientific interests of the project staff include the internal solar rotation (Leibacher 1984; Duvall et al. 1984; Duvall, Harvey and Pomerantz 1986; Harvey 1988; F. Hill 1987; F. Hill et al. 1988); solar cycle frequency changes (Jefferies et al. 1988b); and large-scale flow mapping (F. Hill 1988, 1989, see also the contributed posters from this conference).

In addition to these four major helioseismology networks, at least three limited-duration two-site networks have either existed or are planned. The most successful has been the ESTEC-sponsored Solar Luminosity Oscillation Telescope (SLOT) project, which installed an instrument at Izãna in 1984, and another at Observatorio de San Pedro Mártir, Baja California, Mexico in 1987 (Jiménez et al. 1988b). This two-site network used a four-channel photometer to observe the oscillations in intensity and correlate them with Doppler measurements. This provides information on the adiabatic behavior of the solar atmosphere. Observations with this network have recently ended. Another two-site network has recently begun to obtain non-imaged solar observations with a magneto-optical filter (Cacciani $e t$ al. 1988). The two sites are at Mt. Wilson, Califomia, U.S.A., and at the University "La Sapienza", Rome, Italy. This network began coordinated observations in September of 1988. Finally, a two-site network of imaged observations with a video-magnetograph was attempted by workers at the California Institute of Technology. The two sites were at Big Bear, California, and at Tel Aviv, Israel. Instrumental differences greatly complicated the data merging problem and the attempt was abandoned.

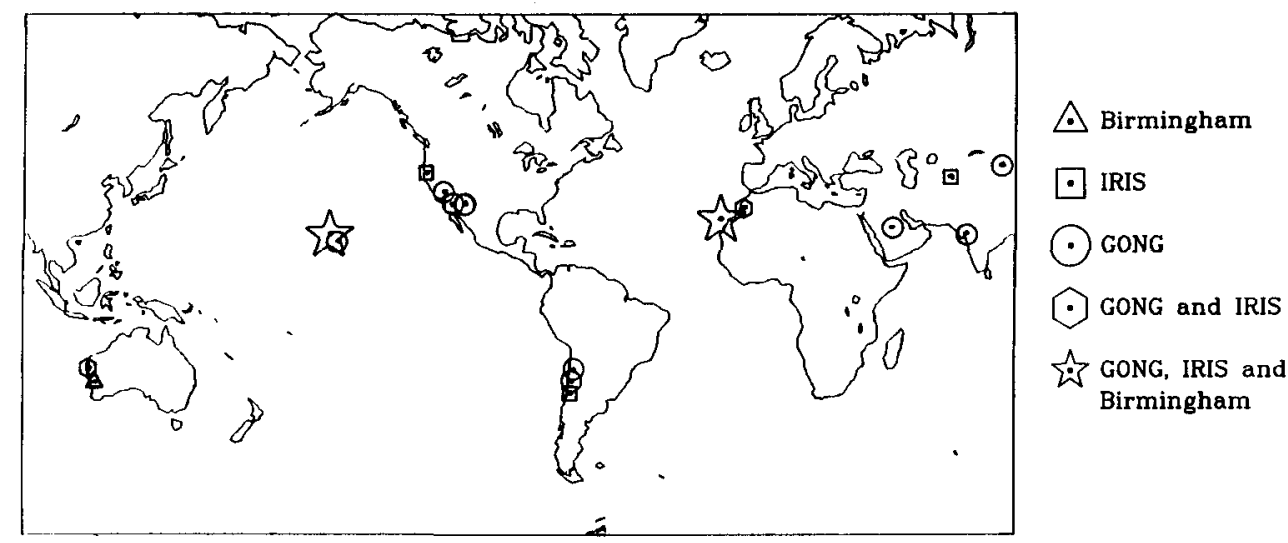

FIGURE 4. A world map showing the actual, planned, or candidate sites for the Birmingham, IRIS, and GONG networks. Note the considerable overlap of the networks, especially in Hawaii and the Canary Islands. This overlap is imposed by the distribution of land on the Earth.

\section{Network Design And Performance - Predicted And Actual}

The main advantage of a network over a single site consists of the reduction of the day/night cycle and the impact of both weather and instrumental failures. In order to maximize this advantage, the location of the sites must be arranged such that at least two and preferably more than two stations are able to potentially observe at any time. If this condition is not met, then the loss of the single station covering a given 
band of Universal Time will result in the immediate reappearance of the diurnal sidelobes in the window power spectrum. The multiple-coverage requirement constrains the choice of locations of the sites, but an even stronger constraint is imposed by the distribution of land on the Earth. The multiple-coverage requirement, along with an average observing day at a single site of eight hours, results in a pattern of six sites, spaced as evenly as possible around the globe. In order to take advantage of the seasonal weather patterns, the six sites should ideally alternate between Northern and Southern hemisphere locations. Perusal of a globe shows that there is virtually no reasonable site in the mid-Atlantic Ocean, forcing one location to be in the mid-Pacific, namely, Hawaii. Once this is realized, then all other longitude bands are set by geography: One must have sites in Australia, mid-Asia, Western Africa, South America, and the Western Coast of North America. These constraints are demonstrated by Figure 4, which shows the locations of the chosen or candidate sites for three of the major networks (Birmingham, IRIS, and GONG) on a map of the world. There is considerable overlap in the choice of sites. Further constraints are imposed scientifically and logistically. Existing, developed astronomical observatories are clearly advantageous, especially if they are solar and have established helioseismology programs. The efficiency of communication and shipping channels are also of concern.

A model of network performance was developed by F. Hill and Newkirk (1985). This model used a simple four-parameter probabilistic description of the temporal distribution of cloud cover at a given site. The most important parameters of the model are the fraction of cloudy time at a site in the summer and winter. For the model, these parameters were estimated from small-scale climatological maps. Such maps cannot reflect micro-meteorological conditions at individual sites that may alter the parameter estimates. The results of the model predicted that a well-chosen six-site network would achieve a duty cycle of about $93.5 \%$.

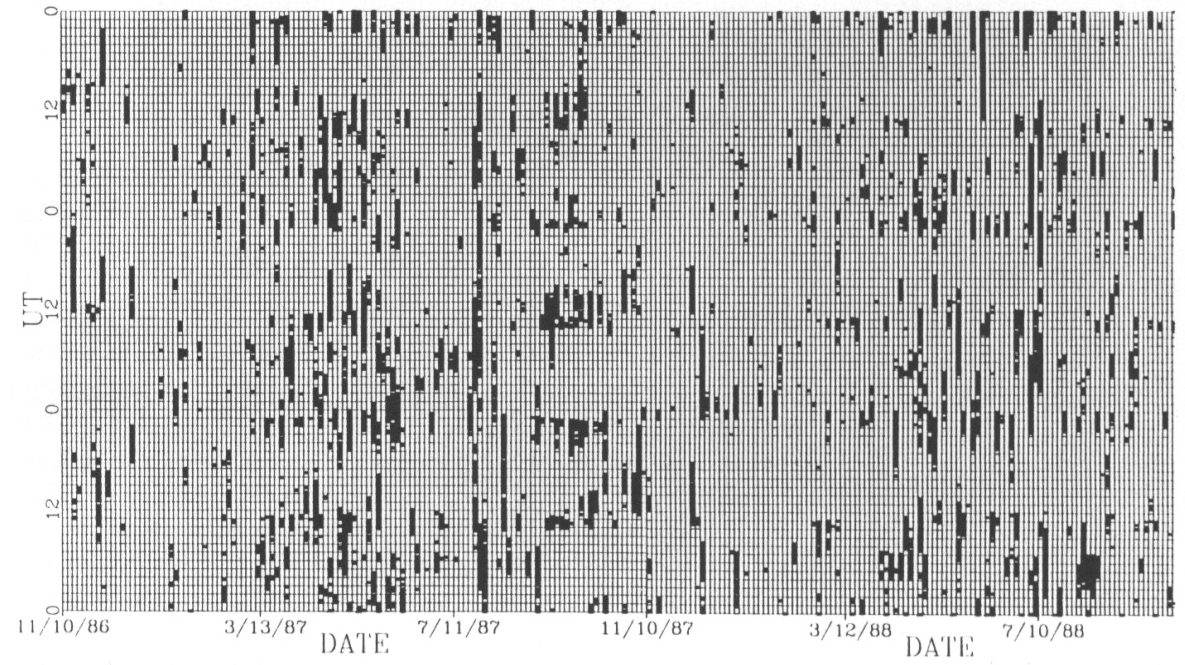

FIGURE 5. The observed window function of one of the candidate six-site GONG networks. Comparison of this window with that in Figure 2 shows the effectiveness of the network in eliminating the diurnal cycle.

The GONG project has been running a network of normal-incidence pyrheliometers since 1985 to measure the parameters and test the model, and to establish communication, shipping, and working relationships with the candidate sites (F. Hill et al. 1988a). Figure 5 shows the actual window function obtained by one of several possible six-site networks in the site survey. Comparison with Figure 2 shows that the diumal cycle has been nearly completely obliterated. The duty cycle obtained by this network is $93.91 \%$, in good agreement with the model prediction. Figure 6 displays the power spectrum of the 
network window function in Figure 5, and shows that the diurnal sidelobes have been greatly attenuated. Comparison of Figure 6 with Figure 3 shows that the amplitude of the fundamental daily sidelobe has been reduced by a factor of 400 , while the combined amplitudes of the first five sidelobes has dropped by a factor of 200 , and the overall background noise power in the first $60 \mu \mathrm{Hz}$ is reduced by a factor of 50 . The longest segment of uninterrupted observations so far achieved by the GONG site survey network is about $\mathbf{4 1 8}$ hours. Thus, the network has fulfilled its design goals, and is capable of producing helioseismic data that is virtually free of contamination by the diurnal sidelobes.

NETWORK $1 \mathrm{~L} / 10 / 86$ OH $0-12 / 28 / 88$ 23H59

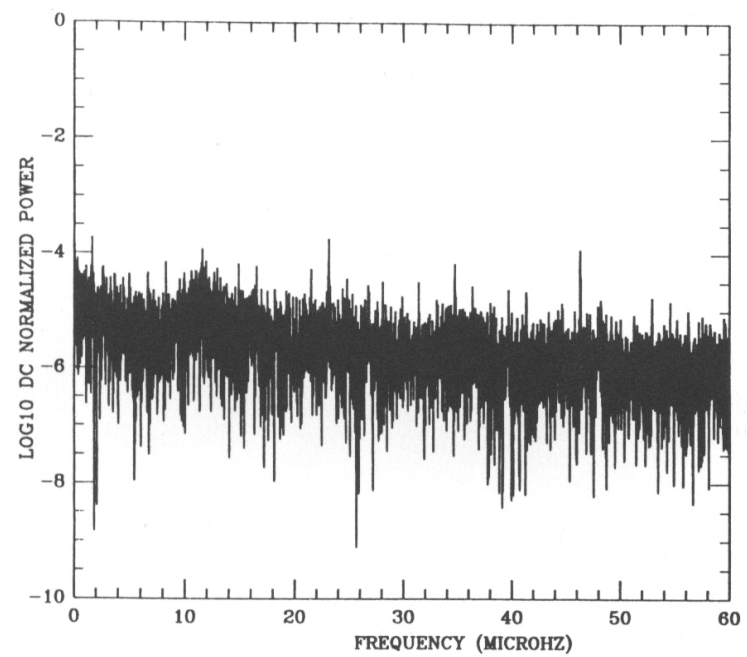

FIGURE 6. The first $60 \mu \mathrm{Hz}$ of the power spectrum of the window function in Figure 5. Comparison of this spectrum with that in Figure 3 shows that the amplitude of the fundamental sidelobe has been reduced by a factor of 400 , the combined amplitudes of the fundamental and first four harmonic sidelobes has been reduced by a factor of 200 , and the overall noise background has been reduced by a factor of 50 .

Figure 7 presents the expected duty cycle of a well-chosen network as a function of the number of sites in the network. The plot shows that the duty cycle rises with the number of sites in the network. It also shows that the dispersion in duty cycle between different site choices decreases with the number of sites. This decrease is also bome out by the results of the GONG site survey. Twelve possible wellchosen networks have so far been examined; they all have duty cycles between 93 and $94 \%$. This is another demonstration of the ability of the network strategy to reduce the effects of weather and instrumental downtime. Figure 7 also plots the actual duty cycles obtained by the GONG site survey for six sites, and the actual duty cycles obtained by the Birmingham network for a few realizations of two and three site networks. The Birmingham results are somewhat lower for the two-site case, and substantially lower for the three-site case. However, there have been logistical problems with the third site that have prevented this version of the Birmingham network from realizing its true potential. Further testing of the model can be done by using the GONG site survey data to form networks with varying numbers of sites; this is in progress.

\section{The Data Merging Problem}

A well-chosen network attempts to have two or more sites observing at all times. The GONG site survey shows that this goal is achieved typically $65 \%$ of the time. Thus, at every temporal step in the observations, two to four simultaneous images will frequently be available. It is clearly desirable to combine 


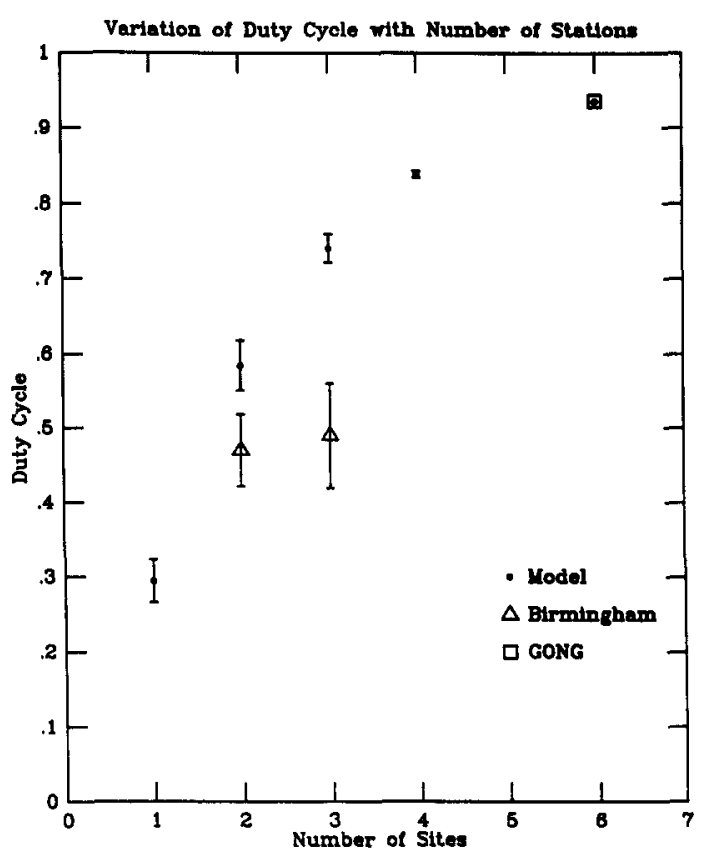

FIGURE 7. The expected duty cycle of a well-chosen network as a function of the number of stations in the network. The theoretical predictions are compared with the actual results from the Birmingham network and the GONG site survey.

these simultaneous images, but a strategy for doing so does not yet exist because of a lack of previous experience. The problem has been faced by the Birmingham network, but it is much simpler to merge a single non-imaged pixel, rather than a set of images. There are many possible strategies, and the final choice will most likely be a combination of approaches matched to the scientific questions being addressed.

The first step is most likely to develop a set of quality assessment measures. Four basic classes of measures can be readily discussed: The first class of global measures concerns the entire image - the rms velocity, intensity, instrumental characteristics, shape of the limb, seeing, scattering, site location, season, zenith angle and transparency variations. The transparency variations are especially important for non-imaged observations because they can easily cause apparent velocity gradients across the entire image in conjunction with the solar rotation gradient (Belmonte $e t$ al. 1988). The second class of quality assessment parameters apply to a single pixel — detector characteristics such as hot pixels, differential stretching distortions, and pixel-sized transparency gradients. The third class of parameters apply to temporal sequences of images, and basically comprise deviations from running means. The fourth class concerns the power spectra of the oscillations, and comprise the amplitudes of the spherical harmonics in various bands of $v$ and $\ell$.

Once these quality measures are determined, it seems intuitively clear that the general answer to the merging problem is a weighted average, but again there are several choices. At what point in the data reduction should the merging be done - before or after the spherical harmonic transform? For instance, seeing effects such as blurring are $\ell$-dependent. How should the weights be chosen - declare one $\ell$-range, pixel, or image better than the others, thereby setting the weights to either 1 or 0 only; or perform a general weighted average of all available images with the weights continuously variable between 1 and 0 ? In 
the latter case, one then has the problem of calculating the weights.

There are issues in the temporal domain as well. What are the merging effects on the temporal power spectra, e.g. since there are six sites in the network, is there any evidence of a four-hour periodicity? There is no strong evidence of enhanced power at this period in the GONG site survey data, but it may be present at a level high enough to affect subtle searches for long-period oscillations such as $g$ modes. Further, discontinuities in the temporal sequence will introduce spurious high frequencies into the spectra.

There are clearly many ideas to explore. Development of the techniques is going on in three directions. The experience of the Birmingham and IRIS networks will provide insight into the non-imaged problem. The GONG site survey data has been used for a preliminary look at the temporal domain effects. The most valuable route for the GONG project involves the use of artificial solar velocity data that has been created and degraded by a set of programs, rather than the Sun, the Earth's atmosphere, and an observing instrument (Hathaway 1988; Global Oscillation Network Group 1987). The advantage of this approach is that the input answer is available to assess the quality of the techniques.

The question of efficient implementation must also be addressed. The GONG data flow will be substantial, and the merging of the data must be done as efficiently as possible. It is thus highly desirable that the process be done automatically, but the nature of the problem may require the application of human judgement, a process that is relatively slow. Artificial intelligence, expert systems, and parallel processing may have to be incorporated into this phase of the GONG data reduction.

\section{Networks And Space Projects}

The combination of both networks and space projects for helioseismology will result in more useful knowledge than either approach alone. The techniques are complementary in some respects, and similar in others. Both aspects are useful: similar observations enable calibration and validation of results, while dissimilar observations can be used to extend the range of data.

The SOHO mission will carry both imaging and non-imaging instrumentation. Non-imaging helioseismology data will be provided by the Global Oscillations at Low Frequencies (GOLF) (Damé 1988) and Variability of solar IRradiance and Gravity Oscillations (VIRGO) (Fröhlich et al. 1988a) instruments on board SOHO, and have already been obtained by the SMM/ACRIM experiment (Woodard and Hudson 1983) and the IPHIR experiment (Fröhlich et al. 1988b). These non-imaging space experiments provide the opportunity for comparison with results from the Birmingham and IRIS networks.

The imaging experiment on SOHO is known as the Solar Oscillations Imager (SOI) (Scherrer et al. 1988), and comprises another variation on the Fourier tachometer technique but using two Michelson interferometers instead of one (Hoeksema et al. 1988). The SOI experiment most closely corresponds to the GONG network. The SOI data with a $1024 \times 1024$ detector will have much higher spatial resolution than the GONG data, and will also be free of atmospheric distortion. This will allow the measurement of the impact of terrestrial atmospheric effects on the GONG data. Care will have to be taken with the telemetry of the SOI data. The bandwidth limits do not allow complete recovery of the entire SOI data stream, forcing the development of on-board processing and data selection. In addition, the use of the Deep Space Network for the telemetry means that the SOI experiment is also a network, but comprising only three sites. While weather is not as great a factor for radio communication as for optical astronomy, the DSN is vulnerable to instrumental and scheduling problems. In addition, the DSN has an uncovered gap during the Northern Hemisphere winter season. The current scheduling of the two projects (SOHO launch in 1995 with a six-year lifetime; GONG observations covering 1993 to 1996) will provide spatially-resolved helioseismology data over a large fraction of the solar cycle. The data for the two experiments will be analyzed and archived together. Thus, the ground-based networks and the spaceborne experiments form a complementary and synergetic approach to helioseismology. 


\section{Summary And Conclusion}

The development of ground-based networks is a fundamental aspect of current work in helioseismology. The networks are an efficient and practical method of greatly reducing the impact of the diurnal rising and setting of the Sun on the power spectra of solar oscillations. The use of networks, in concert with space experiments, holds the promise of greatly increasing our understanding of the inside of the Sun through order of magnitude improvements in the quality of solar oscillation spectra. While this increase is of importance for solar physics, it also bears on aspects of both particle physics and cosmology through the solar neutrino problem. Thus, investment in helioseismology networks will pay handsome scientific dividends.

\section{Acknowledgements}

This paper has benefited from critical readings by John Leibacher, Jack Harvey, and Jim Kennedy. Information on the networks was contributed by Eric Fossat, George Isaak, and Henry Hill.

\section{References}

Many of the references in this list appeared in a few conference proceedings. For compactness, these proceedings will be identified by the location of the conference in the list. The full references are:

Aarhus: Advances in Helio- and Asteroseismology, IAU Symp. 123, ed. J. Christensen-Dalsgaard and S. Frandsen, Dordrecht: Reidel, 1988.

Cambridge: Seismology of the Sun and the Distant Stars, ed. D. O. Gough, Dordrecht: Reidel, 1986.

Snowmass: Solar Seismology From Space, ed. R. K. Ulrich, J. Harvey, E. J. Rhodes, Jr., and J. Toomre, NASA JPL 84-84, 1984.

Tenerife: Seismology of the Sun and the Sun-Like Stars, ed. E. J. Rolfe, ESA SP-286, 1988.

Aindow, A., Elsworth, Y. P., Isaak, G. R., McLeod, C. P., New, R., and van der Raay, H. B. 1988, Tenerife, p. 157.

Anguera Gubau, M., Pallé, P. L., Pérez Hernandez, F., and Roca Cortés, T. 1989, Contributed Papers to Inside the Sun, submitted to Solar Phys.

Bahcall, J. N., and Ulrich, R. K. 1988, Rev. Mod. Phys. 60, 297.

Belmonte, J. A., Elsworth, Y., Isaak, G. R., New, R., Pallé, P. L., and Roca Cortés, T. 1988, Tenerife, p. 177.

Brown, T. M. 1988, Aarhus, p. 491.

Cacciani, A., Rosati, P., Ricci, D., Marquedant, R., and Smith, E. 1988, Tenerife, p. 181.

Claverie, A., Isaak, G. R., McLeod, C. P., van der Raay, H. B., and Roca Cortés, T. 1979, Nature 282, 591.

Claverie, A., Isaak, G. R., McLeod, C. P., van der Raay, H. B., Pallé, P. L., and Roca Cortés, T. 1984, Mem. Soc. Astron. Ital. 55, 63.

Connes, J., and Connes, P. 1984, in Space Research Prospects in Stellar Activity and Variability, ed. A. Mangeney and F. Praderie, Meudon: Obs. de Paris, p. 135.

Damé, L. 1988, Tenerife, p. 367.

Delache, P., and Scherrer, P. H. 1983, Nature 306, 651.

Domingo, V. 1988, Tenerife, p. 363.

Duvall, T. L., Jr., Dziembowski, W., Goode, P. R., Gough, D. O., Harvey, J. W., and Leibacher, J. W. 1984, Nature 310, 22.

Duvall, T. L., Jr., and Harvey, J. W. 1984, Nature 310, 19. 
Duvall, T. L., Jr., Harvey, J. W., and Pomerantz, M. A. 1986, Nature 321, 500.

Elsworth, Y. P., Isaak, G. R., Jefferies, S. M., McLeod, C. P., New, R., Pallé, P. L., Régulo, C., and Roca Cortés, T. 1988a, Tenerife, p. 27.

Elsworth, Y. P., Isaak, G. R., Jefferies, S. M., McLeod, C. P., New, R., van der Raay, H. B., Pallé, P. L., Régulo, C., and Roca Cortés, T. 1988b, Tenerife, p. 535.

Fahlman, G. G., and Ulrych, T. J. 1982, Mon. Not. Roy. Astron. Soc. 199, 53.

Faulkner, J., Gough, D. O., and Vahia, M. N. 1986, Nature 321, 226.

Fischer, G., Hill, F., Jones, W., Leibacher, J. W., McCurnin, W., Stebbins, R. T., and Wagner, J. 1986, Solar Phys. 103, 33.

Fossat, E. 1988, Tenerife, p. 161.

Fossat, E., Gelly, B., Grec, G., and Pomerantz, M. A. 1987, Astron. Astrophys. 177, L47.

Fossat, E., Grec, G., Gavrjusev, V., and Gavrjuseva, E. 1988, Tenerife, p. 393.

Fröhlich, C., Andersen, B. N., Berthomieu, G., Crommelynck, D., Delache, P., Domingo, V., Jiménez, A., Jones, A. R., Roca Cortés, T., and Wehrli, C. 1988a, Tenerife, p. 371.

Fröhlich, C., Bonnet, R. M., Bruns, A. V., Delaboudinière, J. P., Domingo, V., Kotov, V. A., Kollath, Z., Rashkovsky, D. N., Toutain, T., Vial, J. C., and Wehrli, C. 1988b, Tenerife, p. 359.

Garcia, C., Pallé, P. L., and Roca Cortés, T. 1988, Tenerife, p. 353.

Gelly, B., Fossat, E., and Grec, G. 1988, Astron. Astrophys. 200, L29.

Gelly, B., Fossat, E., Grec, G., and Pomerantz, M. 1988a, Aarhus, p. 21.

Gelly, B., Fossat, E., Grec, G., and Schmider, F.-X. 1988b, Astron. Astrophys. $200,207$.

Gelly, B., Grec, G., and Fossat, E. 1986, Astron. Astrophys. 164, 383.

Global Oscillation Network Group 1987, Report \#5: The 1987 Artificial Data Workshop.

Gough, D. O. 1985, Solar Phys. 100, 65.

Grec, G., Fossat, E., and Pomerantz, M. A. 1983, Solar Phys. 82, 55.

Grec, G., Fossat, E., and Vernin, J. 1976, Astron. Astrophys. 50, 221.

Harvey, J. W. 1988, Tenerife, p. 55.

Harvey, J. W., and the GONG Instrument Development Team 1988, Tenerife, p. 203.

Harvey, J. W., Hill, F., Kennedy, J. R., Leibacher, J. W., and Livingston, W. C. 1988, Adv. Space Res. 8, (11) 117.

Harvey, J. W., Kennedy, J. R., and Leibacher, J. W. 1987, Sky \& Telescope 74, 470.

Hathaway, D. H. 1988, Solar Phys. 117, 329.

Henning, H. M., and Scherrer, P. H. 1986, Cambridge, p. 55.

Hill, F. 1987, in The Internal Solar Angular Velocity, ed. B. R. Durney and S. Sofia, Dordrecht: Reidel, p. 45.

Hill, F. 1988, Astrophys. J. 333, 996.

Hill, F. 1989, Astrophys. J. 343, in press.

Hill, F., and the GONG Site Survey Team 1988, Tenerife, p. 209.

Hill, F., Gough, D., Merryfield, W. J., and Toomre, J. 1984, in Probing the Depths of a Star: The Study of Solar Oscillations From Space, ed. R. W. Noyes and E. J. Rhodes, Jr., NASA JPL 400-237, p. 37.

Hill, F., Gough, D. O., Toomre, J., and Haber, D. A. 1988, Aarhus, p. 45.

Hill, F., and Newkirk, G. A. 1985, Solar Phys. 95, 201.

Hill, H. A. 1984, Astrophys. J. $290,765$.

Hill, H. A. 1986, in Neutrino '86: The Twelfth International Conference on Neutrino Physics and Astrophysics, ed. T. Kitagaki and H. Yuta, Singapore: Kim Hup Lee Publishers, p. 221.

Hill, H. A., Bos, R. J., and Goode, P. R. 1982, Phys. Rev. Lett. 49, 1794.

Hill, H. A., Gao, Q., and Rosenwald, R. D. 1988, Tenerife, p. 403.

Hill, H. A., Rabaey, G. F., Yakowitz, D. S., and Rosenwald, R. D. 1986, Astrophys. J. 310, 444.

Hill, H. A., Tash, J., and Padin, C. 1986, Astrophys. J. 304, 560.

Hoeksema, J. T., Scherrer, P. H., Title, A. M., and Tarbell, T. D. 1988, Tenerife, p. 407.

Isaak, G. R. 1986, Cambridge, p. 223. 
Isaak, G. R., Jefferies, S. M., McLeod, C. P., New, R., van der Raay, H. B., Pallé, P. L., Régulo, C., and Roca Cortés, T. 1988, Aarhus, p. 201.

Isaak, G. R., van der Raay, H. B., Palle, P. L., Roca Cortés, T., and Delache, P. 1984, Mem. Soc. Astron. Ital. 55, 91.

Jefferies, S. M., McLeod, C. P., van der Raay, H. B., Palle, P. L., and Roca Cortés, T. 1988a, Aarhus, p. 25.

Jefferies, S. M., Pomerantz, M. A., Duvall, T. L., Jr., Harvey, J. W., and Jaksha, D. B. 1988b, Tenerife, p. 279.

Jiménez, A., Pallê, P. L., Pérez, J. C., Régulo, C., Roca Cort'es, T., Isaak, G. R., McLeod, C. P., and van der Raay, H. B. 1988a, Aarhus, p. 205.

Jiménez, A., Palle, P. L., Roca Cortés, T., Andersen, N. B., Domingo, V., Jones, A., Alvarez, M., and Ledezma, E. 1988b, Tenerife, p. 163.

Kennedy, J. R., and Pintar, J. A. 1988, in Astronomy From Large Databases, ed. F. Murtagh and A. Heck, Garching: ESO, p. 367.

Kuhn, J. R. 1982, Astron. J. 87, 196.

Kuhn, J. R. 1984, Snowmass, p. 293.

Leibacher, J. W. 1984, in Theoretical Problems in Stellar Stability and Oscillations, ed. A. Noels and M. Gabriel, Liège: Institut d'Astrophysique, p. 298.

Morrow, C. A., and Brown, T. M. 1988, Aarhus, p. 485.

Newkirk, G. A. 1980, editor, Solar Cycle and Dynamics Mission, Final Report, NASA Goddard Space Flight Center.

Noyes, R. W., and Rhodes, E. J., Jr. 1984, editors, Probing the Depths of a Star: The Study of Solar Oscillations From Space, NASA JPL 400-237.

Pallé, P. L., Perez, J. C., Régulo, C. Roca Cortés, T., Isaak, G. R., McLeod, C. P., and van der Raay, H. B. 1986, Astron. Astrophys. 170, 114.

Pallé, P. L., Régulo, C., and Roca Cortés, T. 1989, Contributed Papers to Inside the Sun, submitted to Solar Phys.

Pintar, J. A., and the GONG Data Team 1988, Tenerife, p. 217.

Provost, J., and Berthomieu, G. 1988, Tenerife, p. 387.

van der Raay, H. B. 1988, Tenerife, p. 339.

Scherrer, P. H. 1984, Snowmass, p. 173.

Scherrer, P. H. 1986, Cambridge, p. 117.

Scherrer, P. H., Hoeksema, J. T., Bogart, R. S., and the SOI Co-Investigator Team 1988, Tenerife, p. 375.

Schmider, F.-X., Fossat, E., Grec, G., and Gelly, B. 1988, Tenerife, p. 605.

Stebbins, R. T., and Wilson, C. 1983, Solar Phys. 82, 43.

Woodard, M. F. and Hudson, H. S. 1983, Nature 305, 589.

Yerle, R. 1988, Aarhus, p. 87. 